\title{
Mesoscale helicity distinguishes Vinen from Kolmogorov turbulence in helium II
}

\author{
L. Galantucci, ${ }^{1}$ C. F. Barenghi, ${ }^{1}$ N.G. Parker, ${ }^{1}$ and A. W. Baggaley ${ }^{1}$ \\ ${ }^{1}$ Joint Quantum Centre Durham-Newcastle, School of Mathematics, Statistics and Physics, \\ Newcastle University, Newcastle upon Tyne, NE1 7RU, United Kingdom
}

\begin{abstract}
Experiments and numerical simulations show that quantum turbulence exists in two distinct limiting regimes: Kolmogorov turbulence (which shares with classical turbulence the important property of a cascade of kinetic energy from large eddies to small eddies) and Vinen turbulence (which is more similar to a random flow). In this work, we define a mesoscale helicity for the superfluid, which, tested in numerical experiments, distinguishes the two turbulent regimes, quantifying the amount of nonlocal vortex interactions and the orientation of the vortex lines.
\end{abstract}

\section{INTRODUCTION}

Vorticity in superfluid liquid helium (helium II) is not a continuous and unconstrained field, as in ordinary (classical) fluids, but consists of thin vortex lines 1 whose strength (measured by the velocity circulation $\kappa$ ) and thickness (i.e. the vortex core radius $a_{0}$ ) are held fixed by quantum mechanical constraints. In this study we focus on turbulent tangles of vortex lines generated when liquid helium is stirred ${ }^{2}$. 4 . Similar tangles of vortex lines can also be created in trapped atomic Bose-Einstein condensates by laser stirring, by shaking the trap ${ }^{5}$ or by temperature quenches ${ }^{6}$. The natural question is whether this state of disorder (hereafter referred to as quantum turbulence) is similar to turbulence in ordinary fluids (classical turbulence) or not.

The evidence so far is that quantum turbulence can assume two distinct limiting regimes ${ }^{7}[9]$ called respectively Kolmogorov turbulence and Vinen turbulence (see the Section II for a description of their physical properties). The Kolmogorov regime has been observed in helium experiments driven by counter-rotating propellers $\frac{10}{10}$, wind tunnels ${ }^{11}$, towed grids ${ }^{12 \mid 13}$, vibrating grid in ${ }^{3} \mathrm{He}-$ $\mathrm{B}^{14 \mid 15}$, or by the injection of vortex rings $\mathrm{s}^{8}$, and has been reproduced in the numerical simulations of these experiments16/17. At large scales this regime resembles classical turbulence. The Vinen regime (and its crossover to the Kolmogorov regime) has been observed in experiments $s^{8}$ and in numerical simulations $\frac{18}{18}$ of turbulence driven by vortex ring injection; it has also been generated in numerical simulations of helium II turbulence driven by a small heat flux ${ }^{17}$ (also known as counterflow superfluid turbulence), in numerical simulations of turbulence in trapped atomic Bose-Einstein condensates 19 (including the thermal quench of a Bose gas ${ }^{20121}$ ), and in superfluid models of the early universe ${ }^{22}$. This regime seems to have no classical counterpart.

Our aim is to show that, in helium II, Kolmogorov turbulence and Vinen turbulence differ also in terms of what we call mesoscale helicity. Helicity is a property of great importance in classical fluid dynamics, but in the context of quantum fluids its role, and even its definition, are still debated. The mesoscale definition of superfluid helicity which here we propose extends the classical definition of helicity in fluid dynamics to the mesoscale descrip- tion of turbulent vortex lines in helium II provided by the Vortex Filament Model (VFM); the VFM is the best model currently available for turbulent helium II at the non-zero temperatures and length scales typical of most turbulence experiments. Our results shed new light into the different nature of Kolmogorov and Vinen turbulent flows.

The plan of the paper is the following. Firstly we shall review the difference between Vinen and Kolmogorov turbulence regimes (Section II) and recall the mesoscale description of turbulence provided by the VFM (Section III). In Section IV we shall introduce the definition of mesoscale helicity $\mathcal{H}$ for a superfluid. To better understand the physical meaning of $\mathcal{H}$, in Section $\mathrm{V}$ we shall determine $\mathcal{H}$ for simple vortex configurations, before measuring it in two distinct turbulent flows (Section VI). Section VII will discuss how the values of $\mathcal{H}$ distinguish Vinen from Kolmogorov regimes. Section VIII will draw the conclusions.

\section{VINEN VS KOLMOGOROV}

Kolmogorov turbulence ${ }^{23}$ is characterised by the same energy spectrum $\hat{E}(k) \sim k^{-5 / 3}$ observed in classical turbulence in its simplest (homogeneous, isotropic, statistically-steady) form. The energy spectrum $\hat{E}(k)$ describes the distribution of turbulent kinetic energy $E=\int_{0}^{\infty} \hat{E}(k) d k$ over the length scales $2 \pi / k$ (where $k$ is the wavenumber). The $k^{-5 / 3}$ scaling is interpreted as the manifestation of an energy cascade from large to small eddies taking place over the inertial range $k_{D}=$ $2 \pi / D \ll k \ll k_{\eta}=2 \pi / \eta$ where $D$ is the (large) length scale of the energy injection and $\eta$ is the (small) length scale of the viscous dissipation. In quantum turbulence, $k_{\eta}$ must be replaced by $k_{\ell}=2 \pi / \ell$ where $\ell$ is the average distance between the vortex lines ${ }^{24}$; at length scales shorter than $\ell\left(k \gg k_{\ell}\right)$, individual vortex line dynamics (such as Kelvin waves and phonon emission) becomes significant and leads to a departure from classical hydrodynamic turbulence. Numerical simulations ${ }^{25 \mid 26}$ reveal that the $k^{-5 / 3}$ scaling observed for $k \ll k_{\ell}$ in superfluids arises from the partial polarisation of the vortex tangle: vortex lines align locally, forming energy-containing bundles 2728 which can induce flow at large scales. Further evidence 
of classical behaviour arises from the temporal decay of the kinetic energy $E(t)$ and the vortex line density $\mathcal{L}(t)$ which are observed when the forcing which sustains the turbulence in a steady-state is removed 29 31: $E(t) \sim t^{-2}$ and $\mathcal{L}(t) \sim t^{-3 / 2}$, where $\mathcal{L}(t)$ is defined as the vortex length per unit volume at time $t$.

The second limiting form of turbulence, Vinen turbulence, has different signatures. Its energy spectrum $\hat{E}(k)$ lacks the $k^{-5 / 3}$ scaling and may peak at the intermediate scales around $k_{\ell}$ rather than at the large scales $k_{D}$; at larger wavenumbers it displays the characteristic $k^{-1}$ behaviour of an isolated vortex. The last feature suggests that Vinen turbulence is a random-like flow with a weak or absent energy cascade ${ }^{32}$ (indeed the velocity correlation functions become negligible for distances larger than $\left.\ell^{(19 \mid 20}\right)$. If the forcing is removed, Vinen turbulence decays more slowly than Kolmogorov turbulence: $E(t) \sim t^{-1}$ and $\mathcal{L}(t) \sim t^{-1}$. For the sake of completeness, it is important to note that in Vinen turbulence observed in counterflow channels, one-point turbulent velocity statistics (PDFs) are Gaussian (as in classical turbulence) or exhibit quantum peculiar power laws, depending on whether the measurement region, $\Delta$, is $\Delta \gg \ell$ or $\Delta \ll \ell$, respectively 33 .

\section{VORTEX FILAMENT MODEL}

Since our concern is experiments in helium II at nonzero temperatures, we use the Vortex Filament Model (VFM) $)^{36 / 37]}$. The VFM is based on the separation of length scales $a_{0} \ll \ell \ll D$ typical of experiments, where $a_{0}=10^{-10} \mathrm{~m}$ is the vortex core radius, $\ell \approx 10^{-5}$ to $10^{-3} \mathrm{~m}$ is the average distance between vortex lines, and $D \approx 10^{-2}$ to $10^{-1} \mathrm{~m}$ is the system size. In the range of length scales $\ell<\Delta<D$ relevant to turbulence, the VFM describes vortex lines as three dimensional, oriented, reconnecting space curves, $\mathbf{s}(\xi, t)$, where $\xi$ is arclength, carrying one quantum $\kappa$ of circulation. The curves have infinitesimal thickness, unlike quantum vortices described by more microscopic approaches such as the Gross Pitaevskii equation (GPE) 11 or N-body quantum mechanics 38; in other words, the VFM is a mesoscopic model which neglects density variations at the scale of the vortex core. At each time $t$, the VFM describes the vortex tangle as a collection of $N_{v}$ oriented curves of length $L_{i}\left(i=1, \cdots N_{v}\right)$, where $N_{v}, L_{i}$ and the total vortex length $L=\sum_{i=1}^{N_{v}} L_{i}$ vary with $t$. The velocity field $\mathbf{v}(\mathbf{r})$ which all vortex lines induce at a point $\mathbf{r} \neq \mathbf{s}$ (i.e. a point which is not on a vortex line) is given by the Biot-Savart law

$$
\mathbf{v}(\mathbf{r}, t)=\frac{\kappa}{4 \pi} \int_{\mathcal{T}} d \xi \frac{\mathbf{s}^{\prime}(\xi, t) \times[\mathbf{r}-\mathbf{s}(\xi, t)]}{|\mathbf{r}-\mathbf{s}(\xi, t)|^{3}}
$$

where the line integral extends over the entire vortex configuration $\mathcal{T}$ and $\mathbf{s}^{\prime}=\partial \mathbf{s} / \partial \xi$ is the unit tangent vector at the point s. At non-zero temperature, the motion of vortex lines is determined by Schwarz's equation 36 :

$$
\frac{d \mathbf{s}}{d t}=\mathbf{v}(\mathbf{s}, t)+\alpha \mathbf{s}^{\prime} \times \mathbf{w}(\mathbf{s}, t)-\alpha^{\prime} \mathbf{s}^{\prime} \times\left[\mathbf{s}^{\prime} \times \mathbf{w}(\mathbf{s}, t)\right],
$$

where $\mathbf{w}(\mathbf{s}, t)=\mathbf{v}_{n}(\mathbf{s}, t)-\mathbf{v}(\mathbf{s}, t), \mathbf{v}_{n}(\mathbf{s}, t)$ is the velocity of the normal fluid at $\mathbf{s}$, and $\alpha$ and $\alpha^{\prime}$ are temperaturedependent friction coefficients $\sqrt{40}$ accounting for the interaction between normal fluid and vortex lines. Without a core structure, the Biot-Savart integral, Eq. (1), would diverge if evaluated at a point $\mathbf{r}=\mathbf{s}$ on a vortex line, and must be desingularized in a standard way $\sqrt{36}$ which takes into account the vortex core's finite size, $a_{0}$, and the minimum Lagrangian discretization along the vortex lines, $\Delta \xi$. The total superfluid velocity $\mathbf{v}$ of the vortex line at the point $\mathbf{s}(\xi, t)$ can thus be decomposed into near, far and external contributions, i.e. $\mathbf{v}(\mathbf{s}, t)=\mathbf{v}^{f a r}(\mathbf{s}, t)+\mathbf{v}^{\text {near }}(\mathbf{s}, t)+\mathbf{v}^{\text {ext }}(\mathbf{s}, t)$. The far contribution is

$$
\mathbf{v}^{f a r}(\mathbf{s}(\xi, t), t)=\frac{\kappa}{4 \pi} \int_{\mathcal{T}^{\prime}} d \xi^{\prime} \frac{\mathbf{s}^{\prime}\left(\xi^{\prime}, t\right) \times\left[\mathbf{s}(\xi, t)-\mathbf{s}\left(\xi^{\prime}, t\right)\right]}{\left|\mathbf{s}(\xi, t)-\mathbf{s}\left(\xi^{\prime}, t\right)\right|^{3}}
$$

where now the integral extends to all vortex lines present in the tangle (the line through the point $\mathbf{s}$ as well as all other vortex lines) but excludes the neighborhood of the point $\mathbf{s}$ (this is the meaning of the symbol $\mathcal{T}^{\prime}$ ). The near contribution, accounting for this neighborhood, is

$$
\mathbf{v}^{\text {near }}(\mathbf{s}, t)=\frac{\kappa}{4 \pi} \ln \left(\frac{R_{c}}{a_{0}}\right) \mathbf{s}^{\prime} \times \mathbf{s}^{\prime \prime}
$$

Note that $\mathbf{v}^{\text {near }}(\mathbf{s}, t)$ is directed in the binormal direction and (neglecting the dependence on the slow varying logarithmic term) is inversely proportional to the local radius of curvature $R_{c}=1 /\left|\mathbf{s}^{\prime \prime}\right|$ at the point $\mathbf{s}$, where $\mathbf{s}^{\prime \prime}=\partial^{2} \mathbf{s} / \partial \xi^{2}$. Finally the external contribution, $\mathbf{v}^{e x t}(\mathbf{s}, t)$, describes any irrotational flow which is externally applied, for example by bellows or a heater.

\section{MESOSCALE HELICITY}

The classical definition of helicity $\mathcal{H}$ is 41

$$
\mathcal{H}=\iiint_{V} \boldsymbol{\omega}(\mathbf{r}) \cdot \mathbf{v}(\mathbf{r}) d^{3} \mathbf{r}
$$

where $\mathbf{v}$ is the velocity field, $\boldsymbol{\omega}=\nabla \times \mathbf{v}$ is the vorticity field, $\mathbf{r}$ is the position and $V$ is the volume containing the fluid. An ideal fluid evolving without viscosity according to the classical Euler equation conserves both energy and helicity; helicity conservation freezes the flow's topology in time. In real fluids, viscous dissipation destroys both energy and helicity. Helicity is important in turbulence: 
a large value of $\mathcal{H}$ weakens the nonlinearity of the governing Navier-Stokes equation, reducing the direct cascade of energy from large to small length scales느. Spontaneous reflectional symmetry breaking, implying nonzero net helicity, has been reported in turbulent flows with initial and boundary conditions which are symmetric $\stackrel{43 \mid 44}{ }$. Recent work ${ }^{\sqrt{45}}$ has also shown that the interaction of helical modes of the same sign favours three-dimensional inverse energy transfer and the generation of large length scales. In astrophysics, helicity quantifies the lack of mirror symmetry of the flow which favours the generation of magnetic field by dynamo action $\frac{46}{4}$. It is now possible to directly measure the helicity of thin-cored vortices 47 , boosting the interest in the role kinetic helicity plays in constraining the hydrodynamics.

The superfluid component of helium II has zero viscosity and can thus be considered as the closest physical realisation of the mathematical concept of the classical ideal fluid obeying the Euler equation. On the other hand, quantized vortex-lines can reconnect modifying the flow topology which, hence, is not frozen, in contrast to ideal classical fluids. Questions naturally arise: is there an analogue of classical helicity in superfluid helium and other quantum fluids? What is its physical meaning? And, is it conserved?

These issues are currently debated in the context of the Gross-Pitaevskii equation (GPE). In GPE theory ${ }^{1}$, the vortex centerline (the vortex axis) is a nodal line of the wavefunction surrounded by a thin tubular core region of depleted density. Since the GPE vorticity, $\boldsymbol{\omega}$, is a Dirac delta function on the centerline (where the velocity $\mathbf{v}$ diverges), a definition of superfluid helicity based on Eq. (5) requires a careful limiting procedure for $r \rightarrow 0$, where $r$ is the radial distance to the centerline 48 , and raises the question as to whether $\mathcal{H}$, thus defined, is conserved or not. An alternative approach is to define the superfluid helicity using the classical decomposition ${ }^{49}$ of $\mathcal{H}$ for thin vortex tubes into writhe, link and twist, in which case superfluid helicity is zero at all times 50 52. The second approach has a subtle aspect: the twist has an intrinsic component which relies upon the construction of a second vortex line along the vortex centerline in order to define a ribbon; this creates a difficulty in the context of GPE theory where there is only one vortex line in the core, the centerline delta function.

At the mesoscale level of description of superfluid turbulence provided by the VFM, the definition of superfluid helicity should not depend on the detailed nature of the vortex core, provided the definition has not physical or mathematical inconsistencies. Although the GPE is a good quantitative model of gaseous Bose-Einstein condensates, it is only a qualitative model of helium II, which is a liquid, not a weakly interacting Bose gas. A many-body quantum mechanical description of the helium vortex core accounts for rotons $\sqrt{53}$ and a more structured vortex core ${ }^{38}$ than predicted by the GPE, revealing that the the azimuthal velocity has the form of a Rankine vortex with crossover from $v_{\theta} \sim r$ behaviour to $v_{\theta} \sim 1 / r$ behaviour at $r \approx a_{0}$, unlike the vortex solution of the GPE which is $v_{\theta} \sim 1 / r$ for all $r \neq 0$. The vorticity $\boldsymbol{\omega}$ is therefore zero but in narrow tubes of approximately constant cross section $\pi a_{0}^{2}$ with magnitude $\omega=\kappa /\left(\pi a_{0}^{2}\right)$ and direction tangential to the centerline. This result justifies the application of the classical definition of helicity, Eq. (5), to helium II. At the mesoscale level we find (see Appendix 1):

$$
\mathcal{H}(t)=\kappa \sum_{i=1}^{N_{v}} \int_{0}^{L_{i}} d \xi \mathbf{s}^{\prime}(\xi, t) \cdot \mathbf{v}^{n o n}(\mathbf{s}(\xi, t), t),
$$

where $\mathbf{v}^{\text {non }}(\mathbf{s}(\xi, t), t)=\mathbf{v}^{\text {ext }}(\mathbf{s}(\xi, t), t)+\mathbf{v}^{f a r}(\mathbf{s}(\xi, t), t)$ is the non-local velocity $\frac{\sqrt{17}}{1}$ induced at a point $\mathbf{s}$ along a line by distant vortex line elements or by external means.

In order to compare different vortex configurations, the mesoscale helicity $\mathcal{H}$ must be normalized by the vortex length $L$, because, if the helicity density $h(\xi)=$ $\mathbf{s}^{\prime}(\xi) \cdot \mathbf{v}^{n o n}(\mathbf{s}(\xi))$ is constant along the vortex lines, $\mathcal{H}$ would simply be proportional to $L$. If we divide $\mathcal{H}$ by $\kappa L$, we find that the tangle-averaged non-local velocity contribution in the direction of the vorticity is the ratio of mesoscale helicity to the quantum of circulation times the total vortex length, i.e.:

$$
<\mathbf{s}^{\prime} \cdot \mathbf{v}^{n o n}>=\frac{\mathcal{H}}{\kappa L} .
$$

Eq. 77 provides a physical interpretation of mesoscale helicity. We expect therefore that, if the vortex lines are randomly oriented in space (Vinen turbulence), then $\mathcal{H}$ will be approximately zero because the non-local velocity contributions at a point $\mathbf{s}$ on a vortex line will tend to cancel out each others. Vice versa, if the vortex tangle is partially polarised via bundles of quasi-parallel vortex lines within a random tangle (Kolmogorov turbulence), $\mathcal{H}$ will be nonzero. Notice that the existence of large scale flows is not a sufficient condition for large helical flows: for example, two straight parallel vortex lines create a large scale superflow but the total mesoscale helicity is zero.

To test the interpretation of mesoscale helicity outlined in the previous section, we perform numerical simulations using the VFM. In Section V we examine the mesoscale helicity $\mathcal{H}$ of simple vortex configurations. In Section VI we determine $\mathcal{H}$ for two distinct turbulent vortex configurations.

\section{SIMPLE VORTEX CONFIGURATIONS.}

In order to further understand the physical meaning of the mesoscale helicity $\mathcal{H}$ defined in Eq. (6), we numerically compute it for the following simple vortex configurations: (a) a vortex line perturbed by a Kelvin wave; (b) a vortex line perturbed by a Kelvin wave together with a second straight vortex line placed alongside the 
first line and oriented in the anti-parallel direction; (c) a vortex line perturbed by a Kelvin wave together with a second straight vortex line placed alongside the first line and oriented in the parallel direction; (d) a bundle of 13 parallel vortex lines all perturbed by Kelvin waves. The four vortex configurations are displayed in Fig. 1] where vortices are coloured according to the value of the local mesoscale helicity density $h(\xi)=\mathbf{s}^{\prime}(\xi) \cdot \mathbf{v}^{\text {non }}(\mathbf{s}(\xi))$.

In all configurations (a) - (d), the Kelvin wave amplitude is $A=8 \times 10^{-3} \mathrm{~cm}$, its wavelength is $\lambda=0.8 \mathrm{~cm}$, the size of the cubic computational box is $D=\lambda$, the spatial discretization along the vortex lines is $\Delta \xi=$ $1.5 \times 10^{-3} \mathrm{~cm}$ and periodic boundary conditions are employed. In cases (b) and (c) the distance between the perturbed vortex line and the straight vortex line is $d_{\text {lines }}=4 \times 10^{-2} \mathrm{~cm}$, while in case (d) 12 vortex lines are arranged circularly around a central vortex line at distance $d_{K W}=2 \times 10^{-2} \mathrm{~cm}$. As a consequence, given that $d_{\text {lines }} / D=5 \times 10^{-2}$ and $d_{K W} / D=2.5 \times 10^{-2}$ boundary effects can be neglected.

To interpret the resulting values of $\mathcal{H}$, listed in the caption of Fig. 1] it is useful to decompose $\mathcal{H}$ into selfinduced and interaction contributions: $\mathcal{H}=\mathcal{H}_{\text {self }}+\mathcal{H}_{\text {int }}$. The first contribution, $\mathcal{H}_{\text {self }}$, is the sum of the helicity of each vortex line arising from the non-local velocity generated on the line by the line itself (implying that $\mathcal{H}_{\text {self }}=0$ for a single straight vortex line); the second contribution, $\mathcal{H}_{\text {int }}$, is the helicity of the vortex lines due to the nonlocal velocity field generated by the other vortices. The mesoscale helicities of configurations (a), (b) and (c) can therefore be written as $\mathcal{H}^{(a)}=\mathcal{H}_{\text {self }}^{(a)}, \mathcal{H}^{(b)}=\mathcal{H}^{(a)}+\mathcal{H}_{\text {int }}^{(b)}$ and $\mathcal{H}^{(c)}=\mathcal{H}^{(a)}+\mathcal{H}_{\text {int }}^{(c)}$.

We note that a Kelvin wave, arising from an instability of a straight vortex line, has a natural orientation and rotates about the initial unperturbed vortex in direction opposite to the superfluid velocity. We find that when we add an anti-parallel straight vortex line to the perturbed vortex the total mesoscale helicity $\mathcal{H} / \kappa$ increases, while when we add a parallel straight vortex line, the total mesoscale helicity $\mathcal{H} / \kappa$ decreases.

The reason of this behaviour is two-fold. Firstly, $\mathcal{H}_{\text {int }}^{(c)}=-\mathcal{H}_{\text {int }}^{(b)}$ because the local helicity density $h(\xi)$ changes sign as the orientation of the straight line is reversed. Secondly, $\mathcal{H}_{\text {int }}^{(b)}>0$, because in case (b) the line elements of the Kelvin wave which are closer to the straight vortex mutually induce onto each other a positive interaction helicity density $h_{\text {int }}$ whose value is larger than the absolute value of the negative helicity density $h_{\text {int }}$ resulting from the interaction of more distant line elements: the overall sum $\mathcal{H}_{\text {int }}^{(b)}$ is thus positive. More precisely, we find $\mathcal{H}_{\text {int }}^{(b)} / \kappa=2.4 \times 10^{-7} \mathrm{~cm}^{2} / \mathrm{s}$ in case (b) and $\mathcal{H}_{\text {int }}^{(c)} / \kappa=-2.4 \times 10^{-7} \mathrm{~cm}^{2} / \mathrm{s}$ in case (c), leading to the corresponding total values reported in Fig. 1. If we repeat the computation of $\mathcal{H}_{\text {int }}$ for two parallel vortex lines both perturbed by the same Kelvin wave as in (a) and separated by the same distance $d_{\text {lines }}$ as in (b) and (c), we obtain that $\mathcal{H}_{\text {int }} / \kappa=-4.1 \times 10^{-7} \mathrm{~cm}^{2} / \mathrm{s}$. As term of
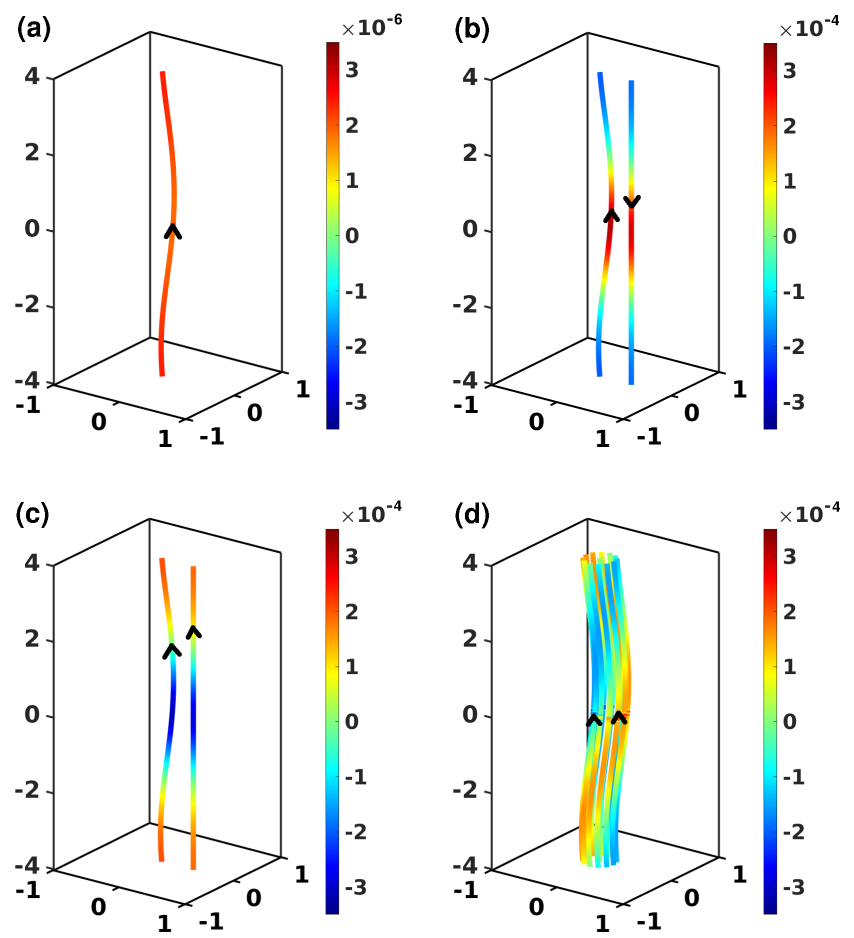

FIG. 1. The four simple vortex configurations (described in the main text) used to illustrate the physical meaning of the mesoscale helicity $\mathcal{H}$ defined in Eq. (6). The computed values of $\mathcal{H}$ are as follows: (a) $\mathcal{H} / \kappa=1.72 \times 10^{-6} \mathrm{~cm}^{2} / \mathrm{s}$; (b) $\mathcal{H} / \kappa=1.96 \times 10^{-6} \mathrm{~cm}^{2} / \mathrm{s} ;$ (c) $\mathcal{H} / \kappa=1.48 \times 10^{-6} \mathrm{~cm}^{2} / \mathrm{s} ;$ (d) $\mathcal{H} / \kappa=-1.10 \times 10^{-5} \mathrm{~cm}^{2} / \mathrm{s}$. Vortices are coloured according to the local value of the helicity density $h(\xi)$. Arrows indicate the orientation of the vortices. The unit of length shown on the axes is $10^{-1} \mathrm{~cm}$.

reference, if in case (a) we employ Eq. (7) to estimate the magnitude of the tangle-averaged non local velocity contribution in the direction of the vorticity $v^{n o n}$, we obtain $v^{n o n}=2.2 \times 10^{-6} \mathrm{~cm} / \mathrm{s}$, which, in this configuration, is significantly smaller than the self-induced velocity along the Kelvin wave $v^{\text {near }}=7.6 \times 10^{-4} \mathrm{~cm} / \mathrm{s}$, computed using Eq. (4) and employing the radius of curvature of the Kelvin wave.

The negative values of $\mathcal{H}_{\text {int }}$ in the circumstance of quasi-parallel vortex lines is the reason why the overall mesoscale helicity $\mathcal{H}$ is negative if the vortex configuration consists of a bundle of Kelvin waves as in Fig. 1 (d). In this configuration, the $i$-th vortex line has a self-induced helicity $\mathcal{H}_{\text {self }}^{i}=\mathcal{H}^{(a)}>0$, hence $\mathcal{H}_{\text {self }}=\sum_{i=1}^{N} \mathcal{H}_{\text {self }}^{i}>0$. However, $\mathcal{H}_{\text {int }}$ is a large negative number because it is the sum of $N(N-1) / 2$ interaction terms which are individually negative (here $N=13$ ). Overall, the total mesoscale helicity $\mathcal{H}=\mathcal{H}_{\text {self }}+\mathcal{H}_{\text {int }}$ is negative.

We conclude that in superfluid turbulence, bundles of quasi-parallel curved vortex lines (which are responsible 
for the existence of large scale flows and quasi-classical Kolmogorov energy spectra ${ }^{28}$ ) possess negative helicity.

\section{TURBULENT VORTEX CONFIGURATIONS}

In the following subsections we determine the mesoscale helicity $\mathcal{H}$ of two distinct turbulent configurations which we refer to as thermally-driven turbulence and injection-driven turbulence. In all numerical simulations of this section, the computational domain is a cube of size $D=1 \mathrm{~cm}$ with periodic boundary conditions. The Lagrangian spatial discretization along the vortex lines is typically $\Delta \xi=1.5 \times 10^{-2} \mathrm{~cm}$ to $2.0 \times 10^{-2} \mathrm{~cm}$, time step is $\Delta t=4 \times 10^{-3} \mathrm{~s}$ to $5 \times 10^{-3} \mathrm{~s}$, and the time evolution is computed using a third order Runge-Kutta scheme. Vortex reconnections $54 \sqrt[56]{56}$ are implemented algorithmically as described elsewhere ${ }^{36157}$ and the Biot-Savart integral Eq. (1) is computed via a tree approximation ${ }^{58}$ in order to decrease the computational cost of the calculations. All simulations start with a few seeding vortex lines which quickly multiply, until, after an initial transient, a statistical steady-state of turbulence is achieved in which the vortex line density $\mathcal{L}$ (or, equivalently, the vortex length $L=\mathcal{L} D^{3}$ ) fluctuates around a mean value independent of the initial condition, as shown by the blue curves in Figures 3 and 5 , for the two experiments. In this steady-state, a balance is reached between forcing and dissipation. Forcing occurs via the Donnelly-Glaberson instability ${ }^{59}$ in thermally-driven turbulence where $T>0$, and via vortex ring injection in injection-driven turbulence; dissipation takes place via the friction in thermallydriven turbulence and via the finite discretization on the vortex lines which models the damping of the shortest Kelvin waves by phonon emission in injection-driven turbulence where $T=0$.

\section{A. Thermally-driven turbulence}

In this numerical simulation, we assume a uniform thermal counterflow velocity $\mathbf{v}_{n s}=\mathbf{v}_{n}-\mathbf{v}^{e x t}$ in Eq. (2), and simulate Vinen turbulence driven thermally by a small heat flux in a large channe ${ }^{17}$ at nonzero temperature. We choose temperature $T=1.9 \mathrm{~K}$ (corresponding to $\alpha=0.206$ and $\left.\alpha^{\prime}=0.009\right)$ and $\left|\mathbf{v}_{n s}\right|=0.08 \mathrm{~cm} / \mathrm{s}$.

In Fig. 2 we report a snapshot of the vortex tangle after the initial transient, where vortex lines are colourcoded according to the local value of the mesoscale helicity density $h(\xi)=\mathbf{s}^{\prime}(\xi) \cdot \mathbf{v}^{n o n}(\mathbf{s}(\xi))$. It emerges that in the Vinen regime the mesoscale helicity density $h(\xi)$ is essentially zero everywhere. This reflects in the temporal evolution of the integrated mesoscale helicity $\mathcal{H}$ divided by $\kappa L$ (reported in Fig. 3), where $\mathcal{H}$ performs small oscillations around zero when the statistically steady-state is achieved.

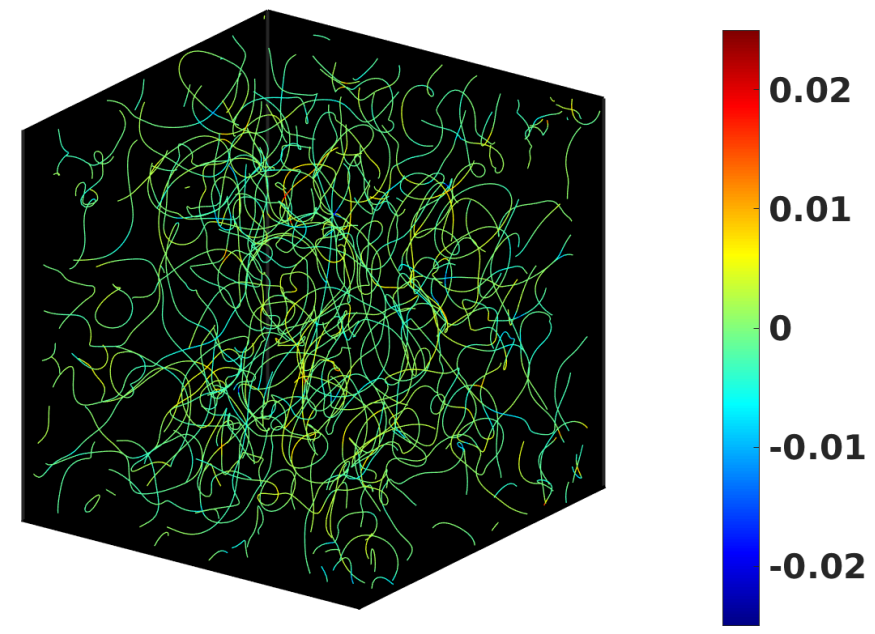

FIG. 2. Thermally-driven turbulence. Snapshot of turbulent vortex tangles driven by uniform normal fluid (Vinen regime) at $t=2400 \mathrm{~s}$. The vortex lines are colour-coded according to the local mesoscale helicity density $h(\xi)=\mathbf{s}^{\prime}(\xi) \cdot \mathbf{v}^{\text {non }}(\mathbf{s}(\xi))$.

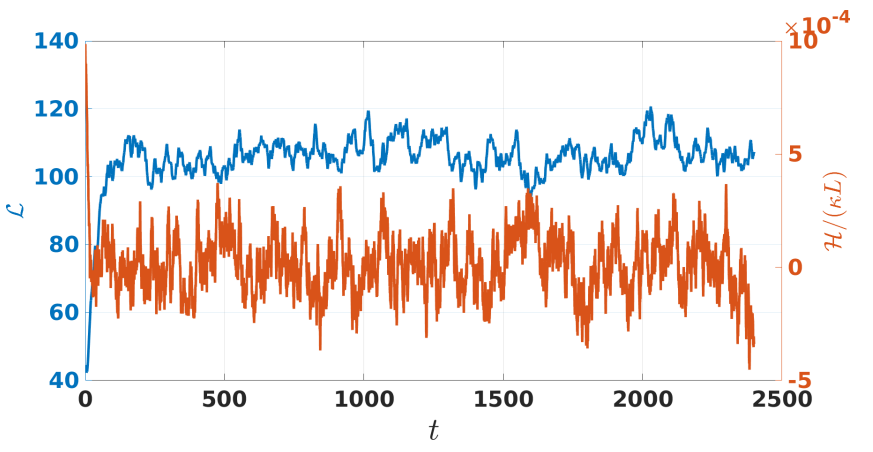

FIG. 3. Thermally-driven turbulence. Temporal evolution of vortex line density $\mathcal{L}(t)\left(\mathrm{cm}^{-2}\right.$, blue curve $)$ and mesoscale helicity $\mathcal{H}$ divided by $\kappa L(\mathrm{~cm} / \mathrm{s}$, red curve $)$ for thermal counterflow. Time $t$ is indicated in seconds.

\section{B. Injection-driven turbulence}

In this numerical experiment we generate turbulence at $T=0$ (no normal fluid) by injecting vortex rings of radius $R=D / 2$ at random positions and with random orientation at the constant rate $\dot{\mathcal{L}}=5.6 \mathrm{~cm}^{-2} / \mathrm{s}(\mathrm{a}$ similar set-up was used in the laboratory by Walmsley and Golov ${ }^{8}$ ). The snapshot of the vortex tangle in the statistically steady-state colored according to the local mesoscale helicity density $h(\xi)$ (Fig. (4) shows that locally $|h(\xi)|$ achieves significantly larger values if compared to Vinen turbulence (Fig. 22). Furthermore, regions with negative $h(\xi)$ seem to prevail over regions with positive $h(\xi)$. This visual suggestion is confirmed by the temporal evolution of $\mathcal{H} /(\kappa L)$ reported in Fig. 5 which shows that, after a transient, $\mathcal{H} /(\kappa L)$ is negative and its magnitude is larger than the one reported for Vinen turbulence (shown in Fig. 3). 


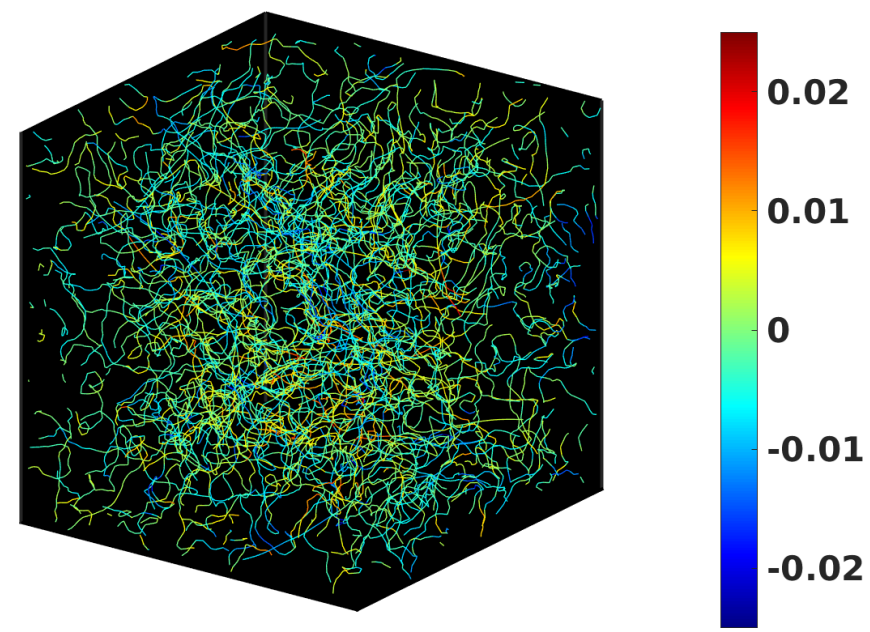

FIG. 4. Injection-driven turbulence. Snapshot of turbulent vortex tangles driven by constant vortex ring injection at zero temperature at $t=175 \mathrm{~s}$. The vortex lines are colour-coded according to the local mesoscale helicity density $h(\xi)=\mathbf{s}^{\prime}(\xi)$. $\mathbf{v}^{\text {non }}(\mathbf{s}(\xi))$.

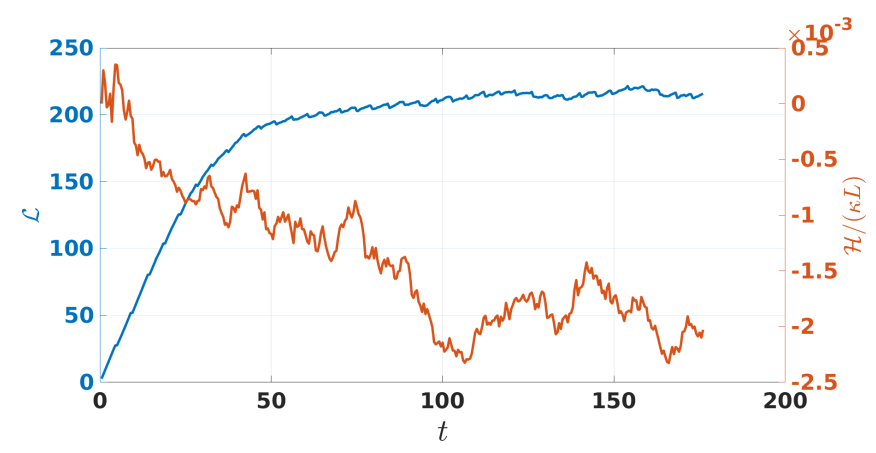

FIG. 5. Injection-driven turbulence. Temporal evolution of vortex line density $\mathcal{L}(t)\left(\mathrm{cm}^{-2}\right.$, blue curve) and mesoscale helicity $\mathcal{H}$ divided by $\kappa L(\mathrm{~cm} / \mathrm{s}$, red curve) for superfluid turbulence driven by constant vortex ring injection at $T=0$. Time $t$ is indicated in seconds.

\section{DISCUSSION}

We interpret the observed behaviours of the magnitude of $\mathcal{H} /(\kappa L)$ in thermally-driven and injection-driven turbulence as follows. In thermally-driven (Vinen) superfluid turbulence the vortex tangle configuration is a random arrangement of vortex loops and lacks large-scale flows. This is confirmed by the kinetic energy spectrum $\hat{E}(k)$ which we report in Fig. 6, which shows the characteristic $\hat{E}(k) \sim k^{-1}$ behaviour of an isolated vortex at $k \approx k_{\ell}$, where $k_{\ell}=2 \pi / \ell$ and $\ell=1 / \sqrt{\mathcal{L}}$ is the average inter-vortex spacing. The absence of large scale flows apparent in figure $\left(\hat{E}(k)\right.$ decreases for $\left.k \rightarrow k_{D}\right)$ implies that the non-local velocity contributions $\mathbf{v}^{\text {non }}$ in Eq. (6) are small (as contributions from distinct vortices tend to cancel out given the random configuration), leading to an overall smaller value of $\mathcal{H} /(\kappa L)$ observed in Fig. 3 with

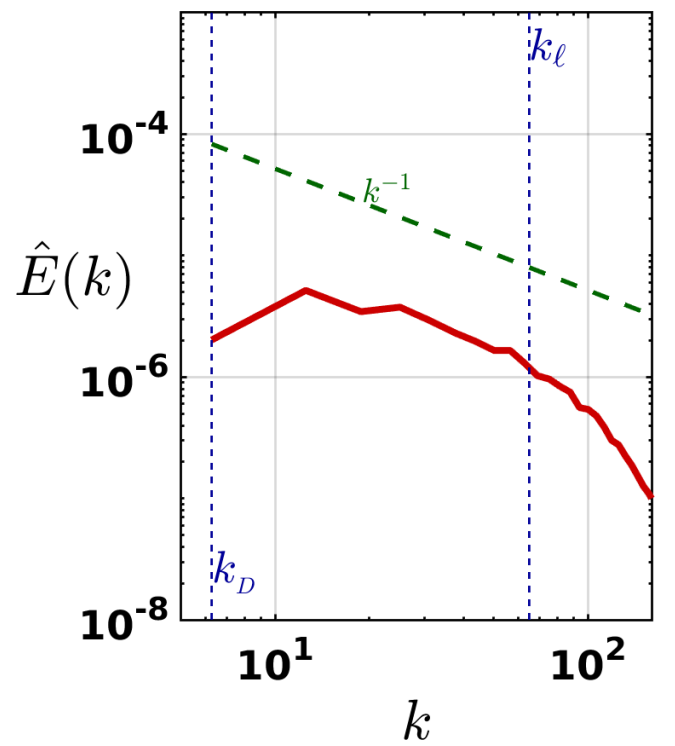

FIG. 6. Thermally-driven turbulence. Counterflow superfluid turbulence at $T=1.9 \mathrm{~K}$ : energy spectrum $\hat{E}$ vs wavenumber $k$ at $t=2400 \mathrm{~s}$; the dashed green line indicates the typical $k^{-1}$ isolated vortex scaling, while blue vertical lines indicate wavenumbers corresponding to the size of the box $D$ and the average intervortex spacing $\ell$.

respect to the value of $\mathcal{H} /(\kappa L)$ calculated for injectiondriven turbulence (Fig. 5).

If the superfluid turbulence is driven by vortex ring injection instead, the energy spectra $\hat{E}(k)$ reveal a quasiclassical Kolmogorov behaviour, with $\hat{E}(k) \sim k^{-5 / 3}$ for $k \ll k_{\ell}$, as shown if Fig. 7. This property implies that most of the energy is contained in large-scale eddies $(\hat{E}(k)$ increases for $k \rightarrow k_{D}$ ). Thus, $\mathbf{v}^{\text {non }}$ is significantly larger than in counterflow turbulence, accounting, see Eq. (7), for the observed larger magnitude of $\mathcal{H} /(\kappa L)$ when compared to thermally-driven turbulence. Furthermore, on the basis of the results presented in Section V, we ascribe the negative value of $\mathcal{H} /(\kappa L)$ in injection-driven turbulence to the presence in the flow of vortex-line bundles which are responsible for the observed Kolmogorov spectrum 2728. For the sake of completeness, in Appendix 3 we report the temporal evolution of mesoscale helicity $\mathcal{H}$ in a numerical simulation of mechanically-driven superfluid turbulence ${ }^{17 / 60}$.

The interpretation of the mesoscale helicity as a measure of the non-local contribution to the motion of the vortex lines, Eq. (7), is confirmed by expressing $\mathcal{H}$ in units of the characteristic self-induced velocity of the vortex lines in the tangle, which can be estimated from Eq. (4) using $R \approx 1 / \sqrt{\mathcal{L}}$. For the thermally-driven turbulence shown in Fig. 2 we obtain $v^{\text {non }} / v^{\text {near }} \approx 0$, as $\mathcal{H}$ fluctuates around zero; the largest fluctuations suggest that $v^{n o n} / v^{n e a r}$ is at most $0.8 \%$. This is consistent with the early finding of Schwarz ${ }^{36}$ that the selfinduced velocity alone is enough to generate the observed intensity of thermally-induced turbulence. For the 


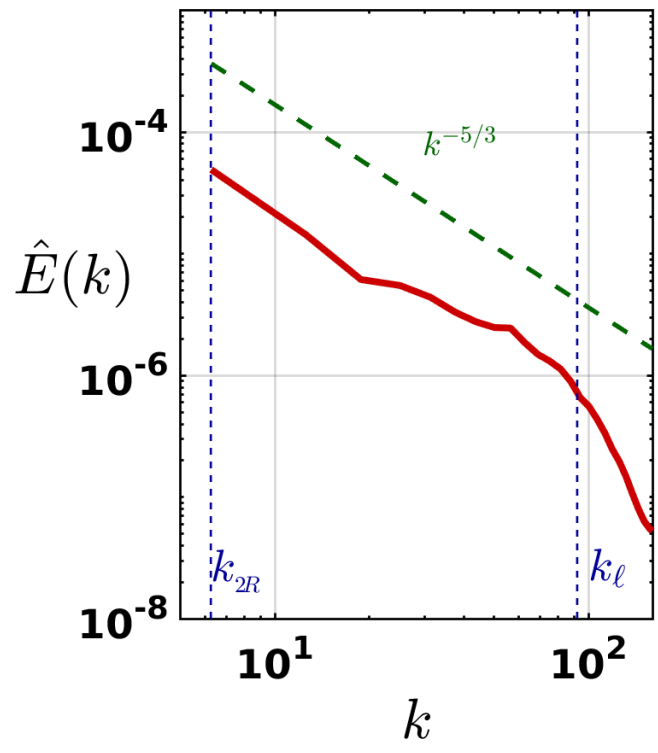

FIG. 7. Injection-driven turbulence. Superfluid turbulence driven by injection of vortex rings at $T=0$ : energy spectrum $\hat{E}$ vs wavenumber $k$ at $t=175 \mathrm{~s}$; the dashed green line shows the slope of the classical Kolmogorov $k^{-5 / 3}$ scaling, while blue vertical lines indicate wavenumbers corresponding to the diameter $2 R$ of the injected vortex rings and the average intervortex spacing $\ell$.

injection-driven turbulence shown in Fig. (4), we obtain $v^{\text {non }} / v^{\text {near }} \approx 11 \%$, a significant contribution to the total velocity, as expected. The model of mechanicallydriven turbulence described in Appendix 3 (Fig. 10) yields $v^{\text {non }} / v^{\text {near }} \approx 1.3$, again as expected as this flow has the Beltrami property of maximal helicity (cf. Appendix 3 for further details).

A final consideration concerns vortex reconnections. The turbulence simulations involve thousands of reconnections. In the VFM, the standard reconnection procedure $\sqrt{57}$ reduces the vortex length (as proxy of energy) to model acoustic losses revealed by more microscopic GPE simulations 6162 . We have therefore carefully monitored $\mathcal{H}$ before, during and after an individual vortex reconnection at zero temperature (no friction). We have found (details are in Appendix 2) that $\mathcal{H}$ is initially conserved, consistently with theoretical ${ }^{63}$, experimenta $\left[\sqrt[64]{6}\right.$ and numerica ${ }^{65}$ studies showing that reconnections do not immediately affect the centerline helicity (helicity of vortex tubes without twist contribution). However, as the reconnection cusp relaxes and Kelvin wavepackets are released (a feature also observed in experiments $\sqrt{66}$ ), the relative proportions of near and far field velocity contributions change, resulting in an overall jump $\Delta \mathcal{H}$. In a turbulent flow, these jumps (being either positive or negative) cancel out in the statistical steady state. It is therefore unlikely that the difference in $\mathcal{H}$ between Kolmogorov and Vinen regimes is caused by the reconnection algorithm, more so since the reconnection rate is larger for Vinen than Kolmogorov turbulence ${ }^{17}$ (polarised vortex lines suffer less reconnections).

\section{CONCLUSIONS}

We have shown that the classical definition of helicity can be naturally generalised to the superfluid mesoscale context of the VFM without inconsistency with the microscopic nature of the helium vortex core. We have also shown that our mesoscale definition of helicity $\mathcal{H}$, Eq. (6), has the remarkable property of quantifying the far-field velocity contributions, $v^{\text {non }}$, which are induced at a point along a vortex line by other vortex lines (or by elements on the same vortex line which are sufficiently far away), see Eq. (7); effectively $\mathcal{H}$ measures the non-local contribution to the vortex motion, which in a random vortex tangle is dominated by the locally-induced velocity, $v^{\text {near }}$.

Our numerical experiments show that in Vinentype turbulence these non-local contributions tend to cancel out (corresponding to vanishing $\mathcal{H} /(\kappa L)$ and $\left.v^{\text {non }} / v^{\text {near }}\right)$, whereas in quasi-classical, Kolmogorov-type turbulence they add up (corresponding to nonzero values of $\mathcal{H} /(\kappa L)$ and $\left.v^{\text {non }} / v^{\text {near }}\right)$, as the tangle becomes polarised and vortex bundles generate large scale flows. Moreover, our numerical simulations with simple vortex structures explain why in Kolmogorov quasi-classical superfluid turbulence, mesoscopic helicity $\mathcal{H}$ is negative, as a result of the presence of such vortex bundles, although larger-scale simulations are needed to rule out the role of any inhomogeneity and anisotropy of the vortex tangle and check how helicity scales with the tangle's size. This helical property of quasi-classical superfluid turbulence is a direct consequence of the concentration of superfluid vorticity in one-dimensional vortex lines: quasiclassical turbulence in superfluids is in fact directly linked to the existence of quasi-parallel vortex bundles which carry negative helicity.

It is also worth emphasising that the presence of nonzero superfluid helicity in turbulence generated by vortex ring injection at $T=0$ demonstrates that helicity is not simply injected in the superfluid flow by the normal fluid.

At the moment the superfluid helicity which we have defined can only be determined in numerical simulations, where, as we have seen, it is a useful monitor of vortex interactions. It is natural to ask what is the outlook for experimental measurements of helicity. Recent experiments on helicity in classical fluid dynamics ${ }^{47}$ show that very slender vortex tubes are required for the experimental visualisation and the interpretation of helicity. Since there is no physical system where vorticity is more concentrated in thin tubes than superfluid helium, the motivation to measure helicity in superfluid helium is clear. A direct laboratory measurement of helicity at cryogenics temperature is not as far-fetched as it may seem. For example, Kelvin waves following superfluid vortex reconnections have been observed in the laboratory $\sqrt{66}$. In order to reconstruct the evolution of the mesoscale helicity, 
what is further needed is a $3 \mathrm{D}$ image of the vortex shape, which is potentially feasible in liquid helium as it is done at room temperature.

\section{ACKNOWLEDGMENTS}

\section{Acknowledgments}

We acknowledge the support of EPSRC grant $\mathrm{EP} / \mathrm{R} 005192 / 1$.

\section{Appendix 1: Derivation of Eq. (6)}

The Rankine-like velocity field observed in the manybody calculations of the helium vortex ${ }^{38153}$ implies that the vorticity $\boldsymbol{\omega}$ is zero everywhere but in a narrow tube of approximately constant cross sectional area $\pi a_{0}^{2}$ along the vortex line (see Figure 8) where its magnitude is $\omega=\kappa /\left(\pi a_{0}^{2}\right)$ and its direction is tangential to the centerline. At time $t$, the vortex configuration consists of a collection of vortices of length $L_{i}\left(i=1, \cdots N_{v}\right)$, and Eq. (1) becomes

$$
\begin{aligned}
\mathcal{H}(t) & =\sum_{i=1}^{N_{v}} \int_{0}^{L_{i}} d \xi \int_{\mathcal{D}_{\xi}} \boldsymbol{\omega}(\xi, \boldsymbol{\sigma}) \cdot \mathbf{v}(\xi, \boldsymbol{\sigma}) d^{2} \boldsymbol{\sigma}= \\
& =\frac{\kappa}{\pi a_{0}^{2}} \sum_{i=1}^{N_{v}} \int_{0}^{L_{i}} d \xi \int_{\mathcal{D}_{\xi}} \mathbf{s}^{\prime}(\xi) \cdot \mathbf{v}(\xi, \boldsymbol{\sigma}) d^{2} \boldsymbol{\sigma}
\end{aligned}
$$

where

$$
\mathbf{v}(\xi, \boldsymbol{\sigma})=\mathbf{v}^{e x t}(\xi, \boldsymbol{\sigma})+\mathbf{v}^{\text {near }}(\xi, \boldsymbol{\sigma})+\mathbf{v}^{f a r}(\xi, \boldsymbol{\sigma}) .
$$

Because of the large separation of length scales between $D, \ell$ and $a_{0}$, both the external superflow and the superfluid velocity field induced by all the other vortices are constant on the disc $\mathcal{D}_{\xi}$ and can be evaluated at $\mathbf{s}(\xi)$. In addition, the typical radius of curvature, $R_{c}$, is always much larger than the vortex core radius: $R_{c} \sim 10^{5} a_{0}$. The neighbourhood $\left|\xi^{\prime}-\xi\right|<\delta$ on the vortex line near a point $\mathbf{s}(\xi)$ is thus effectively straight and perpendicular to the disc $\mathcal{D}_{\xi}$ at distances $\delta$ such that $a_{0} \ll \delta \ll R_{c}$ (range which exists given the huge scale separation characterising the system). At such distances, the superfluid velocity $\mathbf{v}^{\text {near }}(\xi, \boldsymbol{\sigma})$ induced by the closest vortex line elements on $\mathcal{D}_{\xi}$ is perpendicular to the unit tangent vector $\mathbf{s}^{\prime}(\xi)$, yielding zero contribution to $\mathcal{H}$. Concerning the line element centered in $\mathbf{s}(\xi, t)$, the only non-zero contribution to $\mathcal{H}$ which arises from the vortex line going through $\mathbf{s}(\xi, t)$ is the contribution of elements of that line which are sufficiently distant from $\mathcal{D}_{\xi}$, where the induced velocity is constant and can be evaluated at $\mathbf{s}(\xi)$. $\mathcal{H}$ thus reduces to Eq. (6) in the main manuscript:

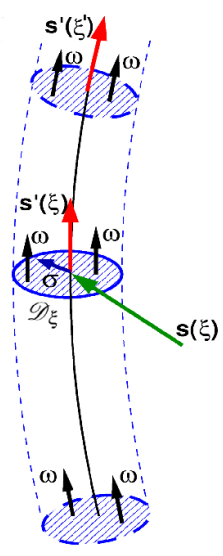

FIG. 8. Schematic vortex tube with cross-sections $\mathcal{D}_{\xi}$ of area $\pi a_{0}^{2}$. The solid (black) line $\mathbf{s}(\xi, t)$ is the centerline. The unit tangent vectors $\mathbf{s}^{\prime}$ at $\mathbf{s}$ are the (red) arrows. The (blue) vector $\boldsymbol{\sigma}$ is the position vector on each cross-section. Within the vortex tube, the vorticity $\boldsymbol{\omega}$ is constant.

$$
\mathcal{H}(t)=\kappa \sum_{i=1}^{N_{v}} \int_{0}^{L_{i}} d \xi \mathbf{s}^{\prime}(\xi) \cdot \mathbf{v}^{n o n}(\mathbf{s}(\xi))
$$

where

$$
\mathbf{v}^{n o n}(\mathbf{s}(\xi))=\mathbf{v}^{e x t}(\mathbf{s}(\xi))+\mathbf{v}^{f a r}(\mathbf{s}(\xi)),
$$

is the superfluid velocity at a point $\mathbf{s}(\xi)$ of a vortex line induced by distant vortex line elements on that line and other lines $\left(\mathbf{v}^{f a r}\right)$, added to the external potential superflow $\mathbf{v}^{e x t}$.

\section{Appendix 2: Helicity during reconnection}

Figure 9 illustrates a single reconnection of two initially orthogonal vortex lines at zero temperature simulated using the VFM in a periodic domain. The reconnection occurs at $t \approx 48 \mathrm{~s}$, where the total length suddenly decreases due to the reconnection algorithm (on a longer time, the vortex lines are slightly stretched). There is a small time delay between the reconnection and the resulting jump $\Delta \mathcal{H}$ of mesoscale helicity caused by the relaxing vortex cusp (notice the Kelvin waves which move away); this jump represents the changed proportion of near and far field velocity contributions. Before and after the reconnection, the mesoscale helicity is constant (there is no friction, unlike the simulations presented in the main paper).

\section{Appendix 3: Mechanically-driven turbulence}

In this appendix, for the sake of completeness given previous studies reported in the literature ${ }^{17 / 60}$, we perform a third superfluid turbulence numerical simulation. In particular, we consider superfluid helium at nonzero 


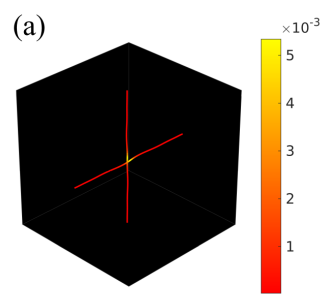

(c)

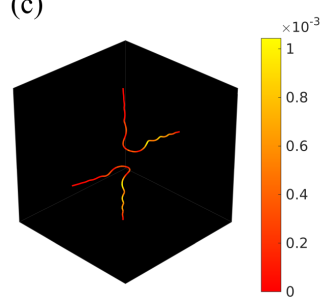

(b)

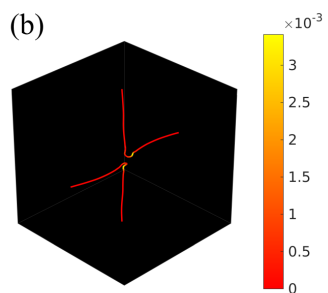

(d)

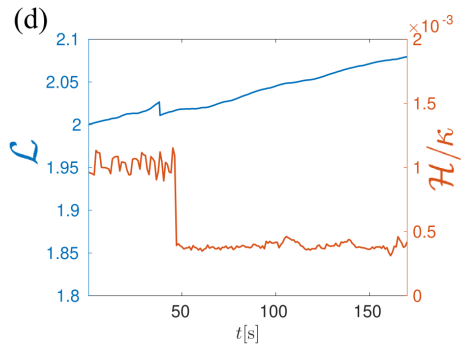

FIG. 9. (a)-(c) Snapshots of two initially orthogonal vortices undergoing a reconnection at $T=0$. Vortex lines are colour-coded according to the local value of the magnitude of the mesoscale helicity density $\left|\mathbf{s}^{\prime}(\xi) \cdot \mathbf{v}_{\text {far }}(\mathbf{s}(\xi))\right|$. (d) Evolution of the vortex line density $\mathcal{L}$ in $\mathrm{cm}^{-2}$ (blue) and volume integrated mesoscale helicity $\mathcal{H} / \kappa$ in $\mathrm{cm}^{2} / \mathrm{s}$ (red).

temperature driven mechanically, e.g. by propellers, which induce turbulence in the normal fluid. In this case, we model the coherent regions of intense normal fluid vorticity typical of classical turbulence by imposing a steady ABC normal fluid flow $1760 \mathbf{v}_{n}=\left(v_{n x}, v_{n y}, v_{n z}\right)$ :

$$
\begin{aligned}
& v_{n x}=A \sin (k z)+C \cos (k y), \\
& v_{n y}=B \sin (k x)+A \cos (k z), \\
& v_{n z}=C \sin (k y)+B \cos (k x)
\end{aligned}
$$

with $A=B=C=0.03 \mathrm{~cm} / \mathrm{s}, k=2 \pi \mathrm{cm}^{-1}$, and temperature $T=1.9 \mathrm{~K}$. The scale of the forcing is the size the computational box $D$. All numerical parameters of the simulation (box-size, vortex-line spatial discretization and time-step) coincide with the parameters illustrated in Section VI.

In Fig. 10 we report a helicity density colored snapshot of the vortex tangle in the steady-state regime: it shows regions of large magnitude of positive helicity density $h(\xi)$, if compared to snapshots of the vortex tangle corresponding to thermally-driven and injection-driven turbulence (Fig. 2 and Fig. 4, respectively). This is reflected in the temporal evolution of $\mathcal{H} /(\kappa L)$ (shown in Fig. 11 which in the statistically steady-state has a large positive value, one order of magnitude larger than the value of $\mathcal{H} /(\kappa L)$ observed in injection generated superfluid turbulence (Fig. 5).

The fact that $\mathcal{H} /(\kappa L)$ in mechanically-driven turbulence is larger than in thermally-driven and injectiondriven turbulence is expected: the $\mathrm{ABC}$ flow has the Beltrami property of maximal helicity i.e. velocity and vorticity of the normal fluid are locally aligned. As the

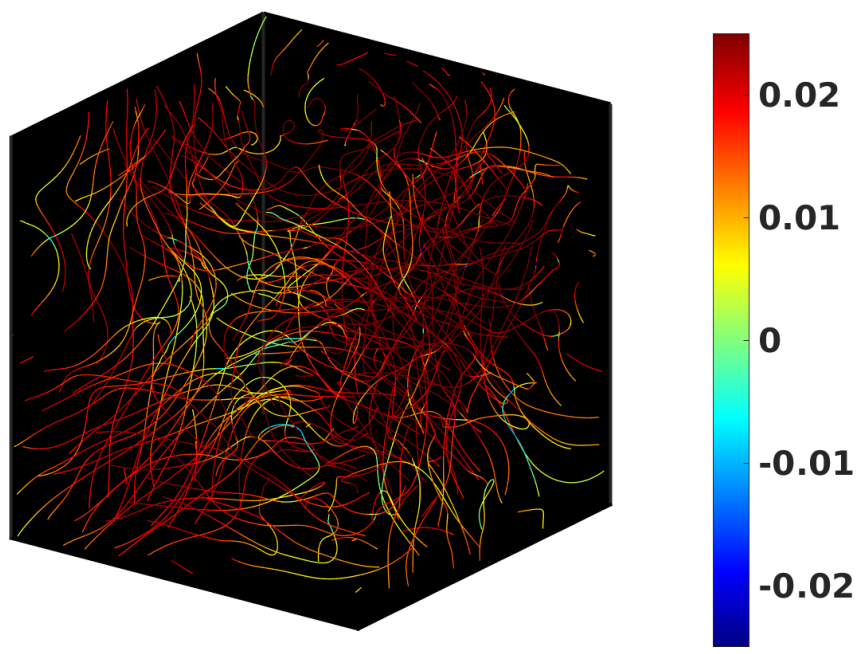

FIG. 10. Mechanically-driven turbulence. Snapshot of turbulent vortex tangle driven by ABC normal flow at $t=2700 \mathrm{~s}$. The vortex lines are colour-coded according to the local mesoscale helicity density $h(\xi)=\mathbf{s}^{\prime}(\xi) \cdot \mathbf{v}^{n o n}(\mathbf{s}(\xi))$.

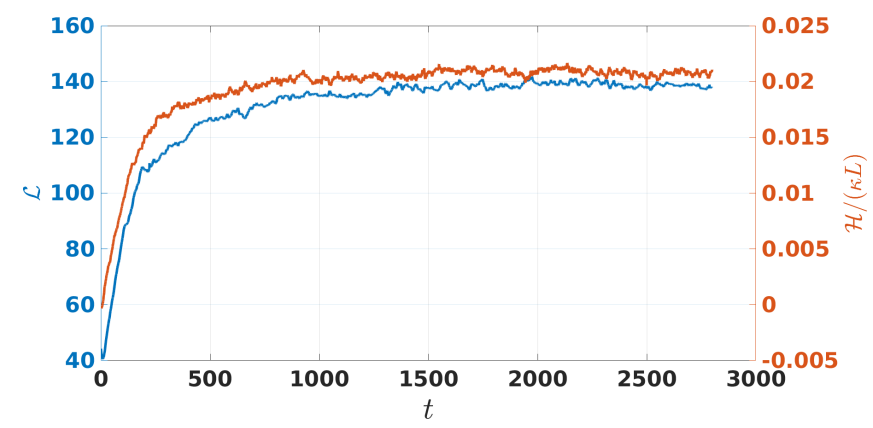

FIG. 11. Mechanically-driven turbulence. Temporal evolution of vortex line density $\mathcal{L}(t)\left(\mathrm{cm}^{-2}\right.$, blue curve) and mesoscale helicity $\mathcal{H}$ divided by $\kappa L(\mathrm{~cm} / \mathrm{s}$, red curve) for superfluid turbulence driven by $\mathrm{ABC}$ normal flow. Time $t$ is indicated in seconds.

imposed normal fluid velocity field, which forces the superfluid turbulence, is stationary, superfluid vortex lines tend to align with the normal fluid vorticity $\sqrt{60}$ boosting the growth rate of helical Kelvin waves arising from the Glaberson-Donnelly instability. It is therefore possible to conclude that in mechanically-driven turbulence the large superfluid mesoscale helicity $\mathcal{H}$ is an effect of the normal fluid via the mutual friction interaction.

This large value of $\mathcal{H}$ is consistent with the computed average magnitude of non-local velocity contributions $\mathbf{v}^{\text {non }}$, in the spirit of Eq. (7). Indeed, if we calculate $\left\langle\left|\mathbf{v}^{\text {non }}\right|\right\rangle$ in the statistically steady-state we obtain $2.1 \times 10^{-2} \mathrm{~cm} / \mathrm{s}$, larger than the corresponding values evaluated for thermally-driven and injection-driven superfluid turbulence, respectively $0.45 \times 10^{-2} \mathrm{~cm} / \mathrm{s}$ and $0.96 \times 10^{-2} \mathrm{~cm} / \mathrm{s}$. We stress that these computed values of $\left\langle\left|\mathbf{v}^{\text {non }}\right|\right\rangle$ for the three numerical simulations of superfluid turbulence only show the the qualitative behaviour of $\mathcal{H} /(\kappa L)$ across the three distinct systems, as in Eq. (7) 


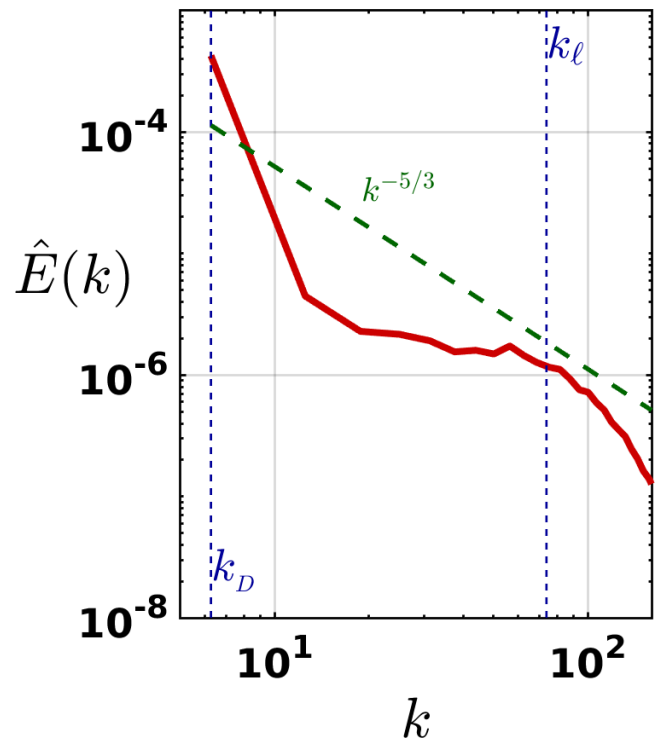

FIG. 12. Mechanically-driven turbulence. Superfluid turbulence driven by $\mathrm{ABC}$ normal flow: energy spectrum $\hat{E}$ vs wavenumber $k$ at $t=2700 \mathrm{~s}$; the dashed green line shows the slope of the classical Kolmogorov $k^{-5 / 3}$ scaling, while blue vertical lines indicate wavenumbers corresponding to the size of the box $D$ and the average intervortex spacing $\ell$. only the tangential component of $\mathbf{v}^{\text {non }}$ is averaged over the vortex tangle. To conclude this section, in Fig. 12 we report the superfluid energy spectrum $\hat{E}(k)$ for this mechanically-driven superfluid turbulent flow. As a result of the stationary forcing imposed at the largest scale of the flow (the box-size $D=1 \mathrm{~cm}$ ), $\hat{E}(k)$ is peaked at the highest wavenumber $k_{D}$, producing a much steeper spectrum.
1 C. F. Barenghi and N. G. Parker, A Primer on Quantum Fluids, Springer (2016).

2 C. F. Barenghi, L. Skrbek, and K. R. Sreenivasan, Proc. Nat. Acad. Sci. USA 111 (suppl. 1), 4647 (2014).

${ }^{3}$ L. Skrbek and K. R. Sreenivasan, Phys. Fluids 24, 011301 (2012).

4 S. K. Nemirovskii, Phys. Reports 524, 85 (2013).

5 E. A. L. Henn, J. A. Seman, G. Roati, K. M. F. Magalhães, and V. S. Bagnato, Phys. Rev. Lett. 103, 045301 (2009).

${ }^{6}$ G. Lamporesi, S. Donadello, S. Serafini, F. Dalfovo, G. Ferrari, Nat Phys 9, 656 (2013).

7 G. E. Volovik, JETP Lett. 78, 533 (2003).

8 P. M. Walmsley and A. I. Golov, Phys. Rev. Lett. 100, 245301 (2008).

9 S. K. Nemirovskii, Low Temp. Phys. 45, 841 (2019).

10 J. Maurer and P. Tabeling, Europhys. Lett. 43, 29 (1998).

11 J. Salort, C. Baudet, B. Castaing, B. Chabaud, F. Davidaud, T. Didelot, P. Diribarne, B. Dubrulle, Y. Cagne, F. Gauthier, A. Girard, B. Hébral, B. Rousset, P. Thibault and P. E. Roche, Phys. Fluids 22, 125102 (2010).

12 M. R. Smith, R. J. Donnelly, N. Goldenfeld, and W. F. Vinen, Phys. Rev. Lett. 71 ,2583(1993)

13 D. E. Zmeev, P. M. Walmsley, A. I. Golov, P. V. E. McClintock, S. N. Fisher, and W. F. Vinen, Phys. Rev. Lett. 115, 155303 (2015).

14 D. I. Bradley, D. O. Clubb, S. N. Fisher, A. M. Guénault, R. P. Haley, C. J. Matthews, G. R. Pickett, V. Tsepelin and K. Zaki, Phys. Rev. Lett. 96, 035301 (2006).

15 D. I. Bradley, S. N. Fisher, A. M. Guénault, R. P. Haley, G. R. Pickett, and V. Tsepelin, Nature Phys. 7, 473 (2011).

16 A.W. Baggaley, C.F. Barenghi, A. Shukurov, and Y.A. Sergeev, Europhys. Lett. 98, 26002 (2012).
17 L.K. Sherwin-Robson, C.F. Barenghi and A.W. Baggaley, Phys. Rev. B 91, 104517 (2015).

18 A.W. Baggaley, C.F. Barenghi, and Y.A. Sergeev, Phys. Rev. B 85, 060501(R) (2012).

19 A. Cidrim, A.C. White, A.J. Allen, V.S. Bagnato, and C.F. Barenghi, Phys. Rev. A 96, 023617 (2017).

20 G.W. Stagg, N.G. Parker, and C.F. Barenghi, Phys. Rev. A 94, 053632 (2016).

21 T. Bland, G.W. Stagg, L. Galantucci, A.W. Baggaley, N.G. Parker, Phys. Rev. Lett. 121, 174501 (2018).

22 P. Mocz, M. Vogelsberge, V.H. Robles, J. Zavala, M. Boylan-Kolchin, A. Fialkov, and L. Hernquist. M.N.R.A.S. 471, 4559 (2017).

23 U. Frisch, Turbulence. The legacy of A.N. Kolmogorov. Cambridge (1995).

${ }^{24}$ C.F. Barenghi, V. L'vov and P.E. Roche, Proc. Nat. Acad. Sci. USA, 111, (Suppl. 1) 4683 (2014).

25 C. Nore, M. Abid, and M.E. Brachet, Phys. Rev. Lett. 78, 3896 (1997).

26 T. Araki, M. Tsubota, and S.K. Nemirovskii, Phys. Rev. Lett. 89, 145301 (2002).

27 A.W. Baggaley, J. Laurie and C.F. Barenghi, Phys. Rev. Lett. 109, 205304 (2012)

28 A.W. Baggaley, Phys. Fluids 24, 055109 (2012).

29 S.R. Stalp, L. Skrbek, and R.J. Donnelly, Phys. Rev. Lett. 82, 4831 (1999).

30 E. Varga, S. Babuin, and L. Skrbek, Phys. Fluids 27, 065101 (2015).

31 J. Gao, W. Guo, and W.F. Vinen, Phys. Rev. B 94, 094502 (2016).

32 C.F. Barenghi, Y.A. Sergeev and A.W. Baggaley, Sci. Reports 6, 35701 (2016). 
33 M. La Mantia and L. Skrbek, Europhys. Lett. 105, 46002 (2014).

34 A.W. Baggaley and C.F. Barenghi, Phys. Rev. E 84067301 (2011).

35 L. Galantucci and M. Sciacca Acta Appl Math 132, 281 (2014).

36 K.W. Schwarz, Phys. Rev. B 38, 2398 (1988).

37 R. Hänninen, and A.W. Baggaley, Proc. Nat. Acad. Sci. USA 111 (Suppl. 1) 4667 (2014).

38 D.E. Galli, L. Reatto and M. Rossi, Phys. Rev. B 89, 224516 (2014).

39 P.G. Saffman, Vortex Dynamics, Cambridge (1993).

40 R.J. Donnelly and C.F. Barenghi, J. Phys. Chem. Ref. Data 27, 1217 (1998).

41 H.K. Moffatt, J. Fluid Mech. 35, 117 (1969).

${ }^{42}$ R.H. Kraichnan, J. Fluid Mech. 59, 745 (1973).

43 E. Levich and L. Shtilman, Phys. Lett. A 126, 243 (1988).

44 M. Kholmyansky, M. Shapiro-Orot and A. Tsinober, Proc. Roy. Soc. A 4572699 (2001).

45 L. Biferale, S. Musacchio and F. Toschi, Phys. Rev. Lett. 108, 164501 (2012).

46 A. Brandenburg and K. Subramanian, Phys. Reports 417, 1 (2005)

47 M.W. Scheeler, W.M. van Rees, H. Kedia and D. Kleckner, Science 357, 487 (2017).

48 P. Clark di Leoni, P.D. Mininni and M.E. Brachet, Phys. Rev. A 95, 053636 (2017).

49 H.K. Moffatt and R.L. Ricca, Proc. Roy. Soc. London A 439, 411 (1992).

50 R. Hänninen, N. Hietala and H. Salman, Sci. Reports 6, 37571 (2016).

51 S. Zuccher and R.L. Ricca, Fluid Dyn. Res. 50, 011414
(2018).

52 H. Salman, Proc. Roy. Soc. A 473, 20160853 (2018).

53 I. Amelio, D.E. Galli and L. Reatto, Phys. Rev. Lett. 121, $015302(2018)$

54 J. Koplik and H. Levine Phys. Rev. Lett 71, 1375 (1993).

55 S. Serafini, L. Galantucci, E. Iseni, T. Bienaime, R.N. Bisset, C. F. Barenghi, F. Dalfovo, G. Lamporesi and G. Ferrari Phys. Rev. X 7, 021031 (2017).

56 L. Galantucci, A.W. Baggaley, N.G. Parker, and C.F. Barenghi, Proc. Nat. Acad. Sci. USA 116, 12204 (2019)

57 A.W. Baggaley, J. Low Temp. Phys. 168, 18 (2012).

58 A.W. Baggaley and C.F. Barenghi Phys. Rev B 84, 020504(R) (2011).

59 M. Tsubota, T. Araki and C.F. Barenghi, Phys. Rev. Lett. 90, 205301 (2003).

60 C.F. Barenghi, G.G. Bauer, D.C. Samuels and R.J. Donnelly, Phys. Fluids 9, 2631 (1997).

61 M. Leadbeater, T. Winiecki, D.C. Samuels, C.F. Barenghi, and C.S. Adams, Phys. Rev. Lett. 861410 (2001).

62 S. Zuccher, M. Caliari, A. W. Baggaley and C. F. Barenghi, Phys. Fluids 24, 125108 (2012).

63 C.E. Laing, R.L. Ricca, and D.W.L. Sumners, Sci. Reports 5, 9224 (2015).

64 M.W. Scheeler, D. Kleckner, D. Proment, G.L. Kindlmann, and W.T.M. Irvine, Proc. Natl. Acad. Sci. USA 111, 15350 (2014).

65 S. Zuccher and R.L. Ricca, Phys. Rev. E 95, 053109 (2017).

66 E. Fonda, D.P. Meichle, N.T. Ouellette, S. Hormoz, and D.P. Lathrop, Proc. Nat. Acad. Sci. USA 111, suppl. 1, 4707 (2014). 